\title{
Looking for signatures of sex-specific demography and local adaptation on the $\mathrm{X}$ chromosome
}

\author{
Evelyne Heyer* and Laure Segurel \\ See related research article: http://genomebiology.com/2010/11/1/R10
}

\section{Abstract \\ A population study comparing X-linked variation with that of the autosomes reveals that social organization has a clear impact on X chromosome genetic diversity and that the $\mathrm{X}$ chromosome shows a signature of local adaptation.}

The genetic diversity of humans results from the interplay of three main types of evolutionary force. Mutation and recombination act directly on the genome and produce changes. These genetic changes may then be subject to selection, which can take different forms and leave various signatures locally on the genome. Finally, demographic forces such as migration and genetic drift affect the genome as a whole. Untangling the extent to which each of these forces has an impact on our genomic diversity is the focus of human evolutionary genetics.

Thanks to the large amount of data now available, the number of studies focusing on this problem has grown exponentially over the past few years. A new and promising approach, used by Casto et al. [1] in a study published recently in Genome Biology, is to compare the genetic diversity of the $\mathrm{X}$ chromosome with that of the autosomes. This approach can reveal the importance of both demography and selection in shaping human genetic diversity. The $\mathrm{X}$ chromosome undergoes more drift than autosomes, as its effective population size $\left(N_{\mathrm{e}}\right)$ is threequarters that of autosomes. The $N_{e}$ for any population represents the size of an idealized, randomly mating population that experiences the same amount of genetic drift as the one under study. In particular it assumes an equilibrium sex ratio, a variance of reproductive success equal to 1 and no fluctuation of population size over time. The $\mathrm{X}$ chromosome is also asymmetrically

*Correspondence: Evelyne Heyer. Email: heyer@mnhn.fr Human Population Genetics Team, UMR 7206 EcoAnthropologie MNHN/CNRS/ UPD, 57 rue Cuvier, 75005 Paris, France influenced by male and female demographies, and it is strongly affected by selection, as recessive mutations are very efficiently exposed to selection in haploid males. The $\mathrm{X}$ chromosome therefore has the potential to reveal new aspects of the evolution of our genome.

\section{Demographic factors influencing genomic diversity}

The first task of population genetics is to describe the distribution in the human genome of that part of our genetic diversity that arises solely from the interplay of the genomic and demographic evolutionary forces noted earlier, and which can be considered 'neutral' - that is, not primarily the result of selection. Once we know this neutral distribution, we can try to detect those parts of the genome that do not fit the neutral model and that result from selection. On a worldwide scale, genomic regions of neutral diversity can be characterized by a low level of genetic difference among populations, a decrease in genetic diversity the further a population is from Africa, and a good global correlation between genetic and geographical distances. Another aspect is that men and women have different demographic histories, which creates contrasted levels of genetic differentiation among populations in different parts of the neutral genome according to their sex-specific mode of inheritance (as explained below).

Studies on sex-specific demographic history have traditionally used two uniparental markers: mitochondrial DNA (mtDNA), which is inherited solely via the egg, tells the maternal history, and the Y chromosome, inherited solely via the sperm, tells the paternal history. These markers give us access to gender-specific patterns of genetic diversity. In this context, several studies have highlighted the influence of different social systems (exogamous versus endogamous, patrilocal versus matrilocal, or patrilineal versus matrilineal) on the repartition of the genetic diversity of these two markers. Exogamous or endogamous societies are characterized by a choice of the spouse from outside or within the population, respectively. Patrilocal or matrilocal societies are defined by both spouses living in the husband's or wife's population, respectively. Patrilineal or matrilineal societies 
depend on whether individuals belong respectively to the subgroup of their father or their mother. Indeed, any combination of these social organizations can be found among human societies, and they influence both the sexspecific migration rates of individuals and the sex-specific $N_{e}$, both of which in turn have an impact on the level of genetic differentiation between populations. For example, in a patrilocal society the $\mathrm{Y}$ chromosome is more highly differentiated (compared with other populations) than is the mtDNA; conversely, in a matrilocal society the level of differentiation is higher for mtDNA [2,3]. Patrilineal social organization also shapes Y-chromosome differentiation [4].

One of the limitations of studies using these uniparental markers is that they cannot untangle the effect of drift (measured by $N_{\mathrm{e}}$ ) from the effect of migration (measured by the migration rate). Furthermore, the influence of selection on any genetic change cannot be ruled out. One way of avoiding some of these limitations is to compare the genetic diversity of the $\mathrm{X}$ chromosome with that of the autosomes. Women have two copies of the $\mathrm{X}$ chromosome whereas men have only one; therefore the $\mathrm{X}$ chromosome spends two-thirds of its time in women. Thus, its genetic diversity depends more on female demography than does that of the autosomes, which are symmetrically influenced by male and female demography. This means that comparing these two markers (X chromosomes and autosomes) enables a better understanding of the sex-specific intensity of migration and drift on genetic differentiation, respectively.

An increasing amount of data on the $\mathrm{X}$ chromosome has emerged over recent years. One of the first studies comparing the genetic diversity of the $\mathrm{X}$ chromosome and the autosomes concluded that no gender-specific demography was needed to explain the observed level of diversity [5]. This conclusion contrasted with previous conclusions obtained using different markers - that there had been a higher migration rate for women compared with men (see [6] for a review). Since then, other studies have provided contradictory results about the comparative level of genetic diversity on the autosomes and the $\mathrm{X}$ chromosome. While Hammer et al. [7] found a higher effective size for the $\mathrm{X}$ chromosome as compared with the autosomes, which is primarily interpreted as revealing a high variance in reproductive success in males, Keinan et al. [8] revealed a more intense drift on the $\mathrm{X}$ chromosome than expected, which was interpreted as a higher migration rate for men or a longer generation time for women. At a more local scale, Segurel et al. [9] found a higher $N_{\mathrm{e}}$ for women in some populations of Central Asia, which was interpreted to be the consequence of their specific patrilineal social organization, where men who are closely paternally related are clustered in the same population. Therefore, the comparative demography of males and females, as well as the importance of drift in forming genetic diversity in humans, is still largely under debate.

Interestingly, Casto et al. [1] propose that there is no absolute answer to these questions. They studied the 16,297 X-linked single nucleotide polymorphisms (SNPs) genotyped in the CEPH human genome diversity project, which consists of 656,995 biallelic SNPs genotyped in 938 individuals from 51 populations from all over the world. They compared the level of population differentiation of $\mathrm{X}$-chromosome markers with a comparable number of SNPs on an autosome (chromosome 16), and showed that, at a regional scale, the results are different from what is found at a worldwide scale. Worldwide, the withinpopulation diversity of the $\mathrm{X}$ chromosome is reduced compared with that of autosomes, a result compatible with equal migration rates and population size between sexes. But in Eurasia, the within-population diversity for the X-linked and autosomal markers are nearly the same, and in East Asia it is even higher for the X chromosome as compared to autosomes, which could be explained by a higher female $N_{\mathrm{e}}$ in these populations. Their study therefore highlights the fact that the picture of male versus female demography is complex and that there are contrasted histories in different geographical areas, and strengthens the necessity to work not only on a world scale but also on well defined populations at a limited geographical scale.

These studies on the neutral part of our genome are important for two reasons. First, they demonstrate the influence of cultural factors such as social organization on the genetic diversity of our species. Second, they give an estimate of the extent of drift on the evolution of our genome and on the potential for selection to act. The smaller the $N_{\mathrm{e}}$, the larger is the effect of drift and the less will selection shape the genome diversity. The sexspecific demographic history must therefore be taken into account when trying to detect that part of the genome that can be under selective pressure.

\section{Distinguishing selection in the human genome}

The importance of selection in shaping human genomic diversity has been the focus of many studies. The earliest studies were mainly based on interspecific comparisons, such as those between humans and chimpanzees. More recently, huge amounts of data that describe intraspecific human genetic diversity have become available and we are beginning to be able to distinguish the 'neutral' parts of the genome. In turn, this enables us to search for parts of the human genome that are most different between different populations, and thus show evidence of different selective pressures. Such differences are taken as a signature of local adaptation (see, for example $[10,11]$ ). 
Genome-wide studies such as those of Casto et al. [1] are interesting for several reasons. First, they give insights into the importance of local selection in shaping the human genome - that is, the relative importance of our past or recent biological adaptation to different environments. Second, they can reveal new parts of the genome related to local adaptation that could have important biological functions but that have not been the focus of any medical studies so far. Genome scans are therefore very useful for pinpointing regions of the genome of special interest for medical and biological studies.

Chromosome $\mathrm{X}$ is especially dense in genes and so is an interesting target of selection. In the context of sexspecific traits coded for by the $\mathrm{X}$ chromosome, some studies have already shown that allelic frequencies were unevenly distributed among the sexes, and this was interpreted as the consequence of sex-specific selection acting on the $\mathrm{X}$ chromosome [12]. The study of Casto et al. [1] is interesting in this regard, as they are the first to analyze the large set of SNP data available in the CEPH panel specifically focusing on the $\mathrm{X}$ chromosome. Since $\mathrm{X}$-chromosome diversity is strongly shaped by sexspecific behaviors that vary according to social organization, as discussed earlier, any study aiming at the detection of local selection has to be done carefully in order to take these factors into account. The study of Casto et al. [1] is extremely well done in this regard; they first try to explain the differences in $\mathrm{X}$ chromosome and autosome differentiation by sex-specific demography. Then they show that for some parts of the X chromosome, these differences are unlikely to be the result of demographic behavior alone, so they require some selective pressures to be explained. The occurrence of selection is further confirmed by other statistical tests. Finally they pinpoint regions of difference in the $\mathrm{X}$ chromosome, including a $2-\mathrm{Mb}$ region containing a gene that could be the subject of local adaptation - EDA2R, a homolog of the autosomal gene $E D A R$, which codes for hair thickness in East Asia. Immediately upstream of $E D A 2 R$ lies the gene hephaestin $(H E P H)$, which is involved in the uptake of iron from food, and could therefore be linked to adaptation to diet.

From an evolutionary point of view, their approach [1] solves one interesting question. Because of the lower $N_{e}$ of the $\mathrm{X}$ chromosome in comparison with autosomes, the evolution of X-chromosome diversity is expected to be shaped more by drift that by selection. Conversely, any recessive mutation that has either a positive or negative effect will be more prone to selection, because it can be the direct target of selection when carried as a single copy in males. The study of Casto et al. [1] shows that there is indeed a signature of local adaptation on the $\mathrm{X}$ chromosome.

Published: 28 January 2010

\section{References}

1. Casto AM, Li JZ, Absher D, Myers R, Ramachandran S, Feldman M: Characterization of X-linked SNP genotyping variation in globally-distributed human populations. Genome Bio/ 2010, 11:R10.

2. Hamilton $G$, Stoneking M, Excoffier L: Molecular analysis reveals tighter social regulation of immigration in patrilocal populations than in matrilocal populations. Proc Natl Acad Sci USA 2005, 102:7476-7480

3. Oota H, Settheetham-Ishida W, Tiwawech D, Ishida T, Stoneking M: Human mtDNA and Y-chromosome variation is correlated with matrilocal versus patrilocal residence. Nat Genet 2001, 29:20-21.

4. Chaix R, Quintana-Murci L, Hegay T, Hammer MF, Mobasher Z, Austerlitz F, Heyer E: From social to genetic structures in central Asia. Curr Biol 2007, 17:43-48.

5. Ramachandran S, Rosenberg NA, Zhivotovsky LA, Feldman MW: Robustness of the inference of human population structure: a comparison of Xchromosomal and autosomal microsatellites. Hum Genomics 2004, 1:87-97.

6. Wilkins JF, Marlowe FW: Sex-biased migration in humans: what should we expect from genetic data? BioEssays 2006, 28:290-300.

7. Hammer MF, Mendez FL, Cox MP, Woerner AE, Wall JD: Sex-biased evolutionary forces shape genomic patterns of human diversity. PLOS Genet 2008, 4:e1000202.

8. Keinan A, Mullikin JC, Patterson N, Reich D: Accelerated genetic drift on chromosome X during the human dispersal out of Africa. Nat Genet 2009, 41:66-70.

9. Ségurel L, Martínez-Cruz B, Quintana-Murci L, Balaresque P, Georges M, Hegay T, Aldashev A, Nasyrova F, Jobling MA, Heyer E, Vitalis R: Sex-specific genetic structure and social organization in Central Asia: insights from a multi-locus study. PLoS Genet 2008, 4:e1000200.

10. Akey JM: Constructing genomic maps of positive selection in humans: where do we go from here? Genome Res 2009, 19:711-722.

11. Barreiro LB, Laval G, Quach H, Patin E, Quintana-Murci L: Natural selection has driven population differentiation in modern humans. Nat Genet 2008, 40:340-345.

12. Balaresque P, Toupance B, Quintana M, Crouau-Roy B, Heyer E: Sex-specific selection on the human X chromosome? Genet Res 2004, 83:169-176.

doi:10.1186/gb-2010-11-1-203

Cite this article as: Heyer E, Segurel L: Looking for signatures of sex-specific demography and local adaptation on the X chromosome. Genome Biology 2010, 11:203. 